\title{
Macht in Organisationen
}

\author{
Falko von Ameln
}

Macht ist zweifellos ein für Organisationsforschung und Organisationsberatung außerordentlich relevantes Thema: „Few concepts are more crucial or more central than power to the understanding of behavior in organizations" (Fairholm 2009, S. 34). Eine Organisation, deren innere Verfasstheit nicht (auch) auf Macht gründen würde, ist- unabhängig von konkreten Organisations- und Gesellschaftsentwürfen - schlichtweg nicht vorstellbar. Eine Person hat in dem Maße Macht über eine andere Person, in dem sie für diese Person wichtige Ressourcen kontrolliert. So verfügt jede und jeder Vorgesetzte insoweit über Macht als sie oder er Entscheidungen treffen kann, die die Mitgliedschaft, die Entlohnung oder die Arbeitsbedingungen der Mitarbeiter/innen betreffen. Diese in die hierarchische Struktur der Organisation eingewobene Möglichkeit zu sanktionieren oder zu begünstigen ist aber nur eine Form der Manifestation von Macht in Organisationen. Auch Organisationsmitglieder ohne hierarchisch gestützte, formale Macht verfügen über Ressourcen (etwa ihre Arbeitsleistung, Wissen, Informationen, Netzwerkkontakte), die die Organisation benötigt. Dies mag in bestimmten Fällen zu einer Machtbalance führen, in der Regel führt die hierarchisch gestützte Machtausstattung der Führungsrolle aber zu einer Asymmetrie der Kräfteverhältnisse.

Dass sich unabhängig von der formalen Organisation in der Interaktion der Organisationsmitglieder informelle Machtstrukturen herausbilden, die das Geschehen in mindestens ebenso starkem Maße beeinflussen wie die formale Hierarchie, steht allerdings ebenso außer Frage.

Obwohl also das Thema Macht für das Verständnis des Geschehens in Organisationen eine entscheidende Rolle spielt, hat sich die Organisationsforschung bemerkenswert wenig mit diesem Thema befasst. Dieser Umstand ist vielfach damit begründet worden, dass das Sprechen über Macht tabuisiert sei. Bennis sagt man nach, schon vor fast 50 Jahren Macht als ,the organization's last dirty secret“ bezeichnet zu haben. In diesem Sinne schreibt auch Friedberg (1992, S. 40 f.): ,Will man Macht in Organisationen analysieren, so muss man zunächst einmal das Phänomen konkretisieren und enttabuisieren. [...] Ganz im Gegensatz zur legitimen Autorität verknüpft sich mit Macht und Macht-

Online publiziert: 11.04 .2012

(C) VS Verlag für Sozialwissenschaften 2012

F. von Ameln $(\bowtie)$

Norden, Deutschland

E-Mail: von.ameln@gmx.de 
ausübung immer ein Beigeschmack von Machtmißbrauch, Gewalt und anrüchiger Einflussnahme. Kurzum, Macht ist böse, und über sie zu sprechen, mutet fast obszön an“. Dieser recht pauschale Befund verlangt nach einer differenzierteren Betrachtung. Wie die in jüngerer Zeit sehr intensiv geführten gesellschaftlichen Debatten zeigen, wird der Diskurs über Macht, ihre Grundlagen und ihre Grenzen in unserem Kulturkreis keineswegs als obszön betrachtet. Bei Führungskräften im höheren Management ist das Thema Macht - etwa in Coachingprozessen - durchaus kein Tabuthema, sondern wird im Gegenteil als Gestaltungsmittel erlebt. Auch im Verhältnis zwischen den Hierarchieebenen wirkt Macht im Regelbetrieb als Residuum, als Größe, die ohne besondere Aufmerksamkeitszuwendung der Beteiligten wirkt. Je nach theoretischem Standpunkt könnte man sagen: Macht wird erst dann salient, wenn sie eingesetzt wird, um Dissens aufzulösen. Oder: Die Hauptfunktion von Macht besteht darin, dass Dissens gar nicht erst entsteht. Inwieweit sich an Dissens Unmut entzündet, hängt dann davon ab, inwieweit Machteingriffe als legitim wahrgenommen werden. Auf der einen Seite dient hierarchische Macht dazu, die Interessen der Organisation durchzusetzen - durch diesen Bezug auf die Organisationsinteressen legitimiert sich die Macht der Führungsrolle. Auf der anderen Seite stellt die Machtausstattung eines Organisationsmitglieds (nicht nur in Führungsfunktionen) diesem Pressionsmittel zur Verfügung, um Ziele durchzusetzen, für die es im Rahmen der jeweiligen organisationalen Logik keine andere Legitimationsgrundlage gibt. „Dirty secrets" in Bennis Sinne kommen erst in solchen Fällen auf, wenn Macht als persönlich und nicht sachlich motiviert auftritt, somit die Frage nach der Legitimation von Macht im Raum und damit wiederum der Einsatz von Gegenmacht zur Disposition steht.

Das vorliegende Themenheft versteht sich als Beitrag zu einem tieferen Verständnis von Macht in Organisationen. Das Heft wird von einem Grundlagenbeitrag von Günther Ortmann eröffnet. Dieser Beitrag stellt einleitend die Theorien der Macht von Max Weber, Crozier \& Friedberg und Anthony Giddens sowie das Konzept der Mikropolitik vor. Im Anschluss daran beschäftigt sich Ortmann mit einem Teilaspekt, der aber für die Steuerungsfunktion von Macht in Organisationen zentral ist: Im Anschluss an Überlegungen von Jon Elster leitet Ortmann her, dass Macht dort an ihre Grenzen stößt, wo Zustände angestrebt werden, die man nicht direkt intendieren kann. Dies, so Ortmann, begründet eine gewisse Machtlosigkeit der Macht, die in Organisationen zunehmend zu beobachten ist.

Diese These leitet über zu Susanne Webers Analyse des Spannungsverhältnisses von Macht und Gegenmacht in Organisationen. Im ersten Teil des Textes zeigt die Autorin, dass die Diskurse um Innovation, organisationales Lernen, Weiterbildung und Organisationsberatung stets auf „umkämpften Terrain“ stattfinden und weist Perspektiven einer machttheoretisch begründeten Rekonstruktion dieser Diskurse auf. Daran anschließend werden organisationale Macht und Gegenmacht auf bourdieuscher Grundlage erschlossen und Machtdynamiken in organisationalen Feldern diskutiert. Der Beitrag schließt ab mit einem Ausblick auf habitus- (und feld-)reflexive Organisationsberatung.

Nach einer eingehenderen Begriffsbestimmung widmet sich der Beitrag von Friedrich Glasl der Frage, welche Rolle Machtaspekte in Konflikten spielen. Dabei verdeutlicht der Autor anhand seines bekannten Modells der Konflikteskalation, wie sich Macht in verschiedenen Stadien der Konfliktentwicklung ausprägt und die Dynamik vorantreibt. Darauf aufbauend werden Instrumente vorgestellt, die in der Organisationsmediation 
eine Bearbeitung sensibler Machtaspekte ermöglichen. Abschließend stellt Glasl seine „U-Prozedur“ zur Reflexion von Organisationskulturen und ihrer im Hinblick auf den Umgang mit Konflikten förderlichen Gestaltung.

Ein in jüngster Zeit im Hinblick auf die Machtbalance in Organisationen wieder verstärkt diskutiertes Thema ist die Frage, wie der Frauenanteil in Führungspositionen erhöht werden kann. Anna Mucha und Daniela Rastetter haben sich diesem Themenfeld unter der Leitfrage „Was hindert aufstiegsorientierte Frauen daran, nach Macht zu greifen?" genähert. In den Interviews, die die Autorinnen im Rahmen ihrer empirischen Studie mit weiblichen Nachwuchsführungskräften geführt haben, zeigt sich, dass die Bereitschaft zu mikropolitischem Agieren - mehr als die ,reine Aufstiegsmotivation“ einen entscheidenden Faktor für Aufstiegserfolg darstellt. Ausgehend von diesem Befund werden Schlussfolgerungen für die Ausbildung mikropolitischer Kompetenz bei Frauen und Optionen für ein mikropolitisch orientiertes Karrierecoaching diskutiert.

Falko von Ameln und Josef Kramer beleuchten das Verhältnis von Führung und Macht in Organisationen. Ausgehend von einem systemtheoretischen Fundament werden mit der Machtausstattung von Führung verbundene Paradoxien und Dilemmata der Führungsrolle herausgearbeitet. Dabei wird u. a. auf Ortmanns einleitende Überlegungen zu Macht im Zusammenhang mit nichtintendierbaren Zuständen zurückgegriffen: Führungskräfte sind heute mehr denn je darauf angewiesen, dass ihre Mitarbeiterinnen und Mitarbeiter Leistungen erbringen, die mit formaler Macht nicht zu erzwingen sind. An dieser Stelle können Führungskräfte nur wirksam werden, indem sie persönlichen Einfluss geltend machen - Einfluss und Machteinsatz stehen aber, wie der Artikel zeigt, in einem nicht leicht aufzulösenden Spannungsverhältnis zueinander. Der Beitrag schließt mit Empfehlungen für die Ausgestaltung der Führungsrolle.

Als Abschluss dieses Heftes war ein Beitrag zum Thema „,Macht und Beratung “ geplant, der die vorangegangenen Überlegungen aufgreift und auf ihre Implikationen für Veränderungsprozesse hin reflektiert. Ich bedaure es, dass das Heft ohne diesen Beitrag erscheinen muss. Den Schwierigkeiten ebenso wie den Möglichkeiten, die Beraterinnen und Berater um Umgang mit Machtphänomenen in Veränderungsprozessen haben, sollte innerhalb der Beratungsforschung mehr Aufmerksamkeit zuteil werden. Studien haben gezeigt, dass mikropolitische Auseinandersetzungen wesentlich zum Scheitern von Beratungsprozessen beitragen - ein stärker auf Nachhaltigkeit ausgerichtetes Veränderungsmanagement ist daher sowohl auf bessere konzeptuelle Modellierungen von Machtdynamiken im Beratungssystem als auch auf konkrete Gestaltungsmöglichkeiten angewiesen.

\section{Literatur}

Fairholm, G. W. (2009). Organizational Power Politics. Tactics in Organizational Leadership (2.Aufl.). Santa Barbara: Praeger.

Friedberg, E. (1992). Zur Politologie von Organisationen. In W. Küpper \& G. Ortmann (Hrsg.), Mikropolitik. Rationalität, Macht und Spiele in Organisationen (2.Aufl., S. 39-52). Opladen: Westdeutscher. 